\title{
Speed Limit on Change
}

\section{Experiments with a single calcium atom prove that processes of change have a speed limit determined by the rate at which they can dissipate heat.}

\author{
By Philip Ball
}

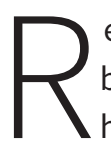
esearchers have verified a general relationship between the rate at which a nonequilibrium process happens-such as the growth of an organism-and the rate at which the process generates entropy [1]. If the entropy production rate is slowed-for example, by some influence that lowers the rate of heat dissipation-then the process takes longer. The team manipulated a single calcium ion with lasers to create a model nonequilibrium system that absorbs and dissipates energy. The result shows what may limit the maximum possible speeds for quantum computation and suggests deep connections between quantum physics and thermodynamics.

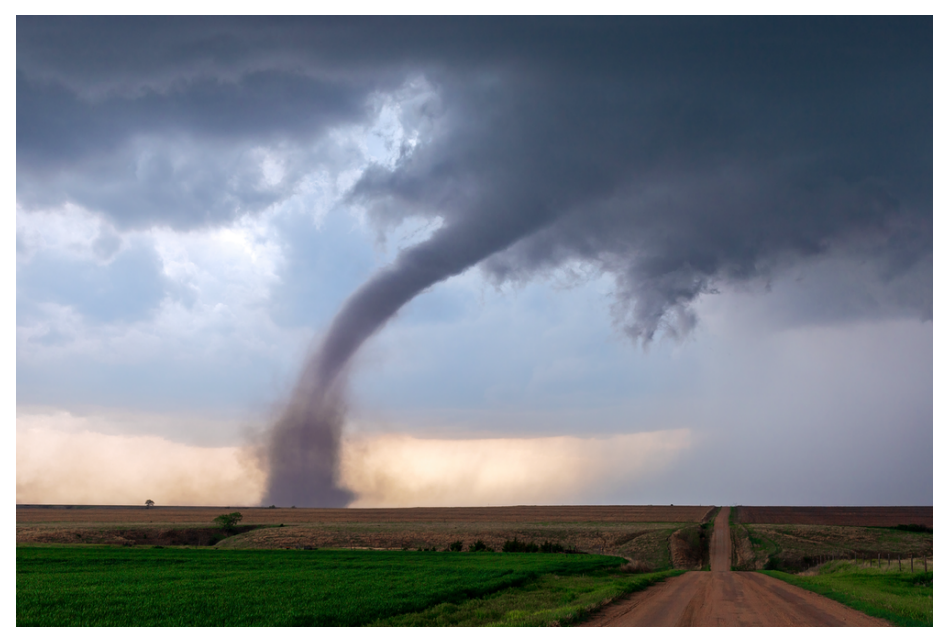

Universal speed limit. A nonequilibrium process like the formation of a tornado, driven by inputs of energy and matter, dissipates energy and generates entropy. According to a new theory, now verified experimentally, the lifetime of such a structure depends on the rate at which it produces entropy.

Credit: JSirlin/stock.adobe.com
Living organisms, the climate system, and human societies are all systems that constantly change but never reach equilibrium, driven by inputs of energy and materials. The second law of thermodynamics states that such changes must increase the total entropy of the Universe.

Entropy production implies that some of the energy driving a nonequilibrium process is converted to heat in the form of random molecular motions that can't be harnessed to do useful work. This conversion to "useless" heat is called dissipation, and it's what makes perpetual motion machines impossible-there is no way to avoid squandering some of the energy driving a device. Making machines and devices run efficiently is partly a matter of minimizing their dissipation.

In 2020, Gianmaria Falasco and Massimiliano Esposito of the University of Luxembourg showed, using the mathematical framework of statistical mechanics, that there is a relationship between the length of time a nonequilibrium process takes $(T)$ and the time-averaged rate of dissipation or entropy production that the process involves $(\langle S\rangle)[2]$. Specifically, the product $\langle S\rangle T$ can never be smaller than Boltzmann's constant $\left(k_{B}\right)$, a fundamental constant of nature.

As a result, for a process to be sped up, its rate of dissipation must increase. This relationship is reminiscent of Heisenberg's uncertainty principle, in which the product of an object's position and momentum uncertainties can never be smaller than Planck's constant, $h$, divided by $4 \pi$. By analogy, Falasco and Esposito called their equation the dissipation-time uncertainty relation. Falasco points out that in the limit of a fully reversible process happening at equilibrium, with zero dissipation, the time taken would be infinite. So in that case, the formula reduces to the well-known result that a reversible change must happen infinitely slowly. 


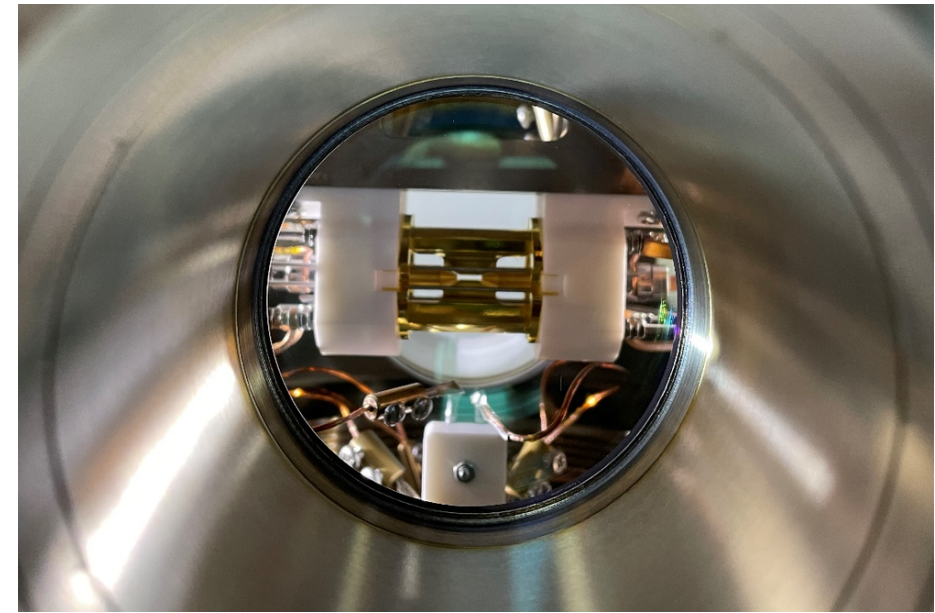

Stuck in the middle. The ion trap used by Feng and co-workers to study the relationship between the lifetimes of excited states of a calcium ion and the rate at which the ion can exchange energy with hot and cold "baths" to enable entropy production.

Credit: M. Feng/APM/CAS

Like all of thermodynamics, the result is expected to apply equally to classical and quantum systems. Mang Feng of the Innovation Academy of Precision Measurement Science and Technology (APM) in China and his colleagues have put the relation to the test in a minimal quantum system: a single calcium ion held in an electromagnetic trap. They selected a quantum version, says Feng, because "nature is basically quantum mechanical, and thus quantum processes are more fundamental."

The team's idea was to stimulate the ion with light and then measure how changes in the dissipation rate affect the time it takes for the ion to switch from one electronic state to another. Such jumps between quantum states happen at random times: all one can predict is the chance of a jump occurring at any moment, but the precise timing of its occurrence is unpredictable. Nonetheless, a formulation of standard quantum theory called quantum-jump theory shows that jumps are governed by dissipation caused by energy exchange between the system and its environment [3]. An energetically excited state of the ion can only decay to a lower-energy state by shedding its excess energy and causing dissipation in the surroundings.
According to the dissipation-time relation, if the rate of dissipation accompanying the decay of an excited state is reduced under some conditions, then the excited state should take longer to decay. But measuring dissipation for a quantum system is immensely difficult because, typically, you can't keep track of all the interactions between the system and its environment-it would be like trying to follow all the collisions of an individual gas molecule. Feng and colleagues made a system where such close monitoring was possible by creating a kind of "artificial environment"-a heat bath-for their trapped ion, which was otherwise carefully isolated from its surroundings and kept very cold.

The researchers used lasers to excite several other electronic states of their ion, in addition to the two involved in the quantum jump. Then the excited state had some probability of shedding or gaining energy by moving along these alternative "dissipative" pathways through the extra quantum states. By carefully adjusting the laser strengths, Feng and colleagues could control the probabilities of the dissipative transitions, such that some dissipative states acted like a "cold" bath that would accept energy from the excited state, and some acted like a "hot" bath that would dump energy into it. In effect, the two-state system, sandwiched between the hot and cold baths, had energy flowing through it, so that it modeled the kind of nonequilibrium dissipative process considered by Falasco and Esposito.

"Our control of the effective two-level system makes it possible to monitor pretty much everything that happens in the quantum evolution of the system plus its 'environment' of [hot and cold] baths," says Feng. This arrangement makes it a kind of "quantum simulator" of a minimal dissipative process.

The researchers were able to track the state of the ion over time and measure both the time $T$ taken to make a jump and the average amount of energy dissipation $\langle S\rangle$ that the jump entailed. Their measurements showed that the quantity $\langle S\rangle T$ could be more than 10 times greater than $k_{B}$ but was never less than $k_{B}$.

Quantum physicist Vlatko Vedral of the University of Oxford in the UK is not surprised that the predicted behavior was confirmed, since he believes the theory may be a variant of a previously recognized relation between time and energy in 
quantum systems. But he says that the experiment is impressive, as it is challenging to keep track of all the energy losses in such a quantum process.

Feng hopes that the results might point to ways of speeding up quantum computation by controlling dissipation. Falasco says that such considerations would also apply to classical electronic devices. But he says that the main applications may be in biology, where similar experiments could, for example, help researchers understand the limitations on the operating speed of molecular motors and other biomolecular "machines."
Philip Ball is a freelance science writer in London. His latest book is The Modern Myths (University of Chicago Press, 2021).

\section{REFERENCES}

1. L.-L. Yan et al., "Experimental verification of dissipation-time uncertainty relation," Phys. Rev. Lett. 128, 050603 (2022).

2. G. Falasco and M. Esposito, "Dissipation-Time Uncertainty Relation,” Phys. Rev. Lett. 125, 120604 (2020).

3. M. B. Plenio and P. L. Knight, "The quantum-jump approach to dissipative dynamics in quantum optics," Rev. Mod. Phys. 70, 101 (1998). 